\title{
LEPTIN AND KISSPEPTIN EVALUATION IN CHILDHOOD OBESITY
}

\author{
F.Leo ${ }^{1}$, A.Mancini ${ }^{1}$, C.Di Segni1 ${ }^{1}$, S.Raimondo ${ }^{1}$, A. Barini², D. Currò ${ }^{3}$, A. Rossodivita ${ }^{4}$
}

\section{Introduction}

Kisspeptin is a neuropeptide secreted in anteroventral-periventricular and arcuate hypothalamic nuclei involved in pubertal onset and in other functions of adult life. Kisspeptin levels are sex dependent, so they are higher in prepubertal girls and in adult women compared to age-matched male. This sex-related pattern has been reported in adult obesity, but few data are reported in childhood obesity. Recent data in knockout mice for Kisspeptin receptor showed increased oxidative stress suggesting also a role of the hormone in metabolic modulation.

We have therefore studied a group of pre-pubertal obese children in order to evaluate correlations between nutritional status, as indicated by Leptin levels, and kisspeptin concentrations.

\section{Methods}

We studied a population of 27 prepubertal children (13 males) aged 5-12 years, classified as overweight $(n=3$, BMI 21,9 \pm 0,98 ; SDS BMI $1,77 \pm 0,06$ ) or obese ( $n=24$ ) (BMI 25,32 \pm 0,89 , SDS BMI $1,94 \pm 0,10$ ) according to Cole's criteria. Eight normal weight children, aged 6-12 years, were enrolled as controls. Several metabolic parameters were evaluated: glucose and insulin levels after oral glucose load, total- LDLand HDL-cholesterol, triglycerides, uric acid, total proteins, C Reactive Protein. Leptin was evaluated using ELISA method (DRG Instruments $\mathrm{GmbH}$, Germany). In order to evaluate kisspeptin levels, morning blood samples were collected; after acidification, peptides were extracted in a C-18 SEP-Column (Phenomenex Inc, USA). The eluted samples were evaporated and stored at $-80^{\circ} \mathrm{C}$ until assayed. Kisspeptin $(\mathrm{pg} / \mathrm{ml})$ was measured using the RIA kit KISS1(61-121)-AmideMetastin(1-54)-NH2 (Phoenix Pharmaceuticals, Burlingame, USA).

\section{Results}

We did not find significant differences in Kisspeptin levels between obese and normal weight children (mean \pm SEM: $20.1 \pm 1.96$ and $20.7 \pm 0.68 \mathrm{pg} / \mathrm{ml}$, respectively) and between males and females obese children $(19.05 \pm 3.39$ and $20.9 \pm 2.49$ $\mathrm{pg} / \mathrm{ml}$ ). Kisspeptin levels did not correlate with SDS BMI, HOMA index and Insulin peak levels after glucose load.

As expected, Leptin levels were higher in obese children than in controls $(20.41 \pm 2.84$ and $4.21 \pm 2 \mathrm{ng} / \mathrm{ml}$, respectively, $\mathrm{p}<$ $0,05)$, while showed a significant correlation with Kisspeptin levels in normal children $\left(R^{2}=0.44 ; p\right.$-value $\left.=0.05\right)$ (Fig. 1$)$, but not in obese $\left(R^{2}=0.03 ; p\right.$-value $\left.=0.6\right)$ (Fig. 2$)$.

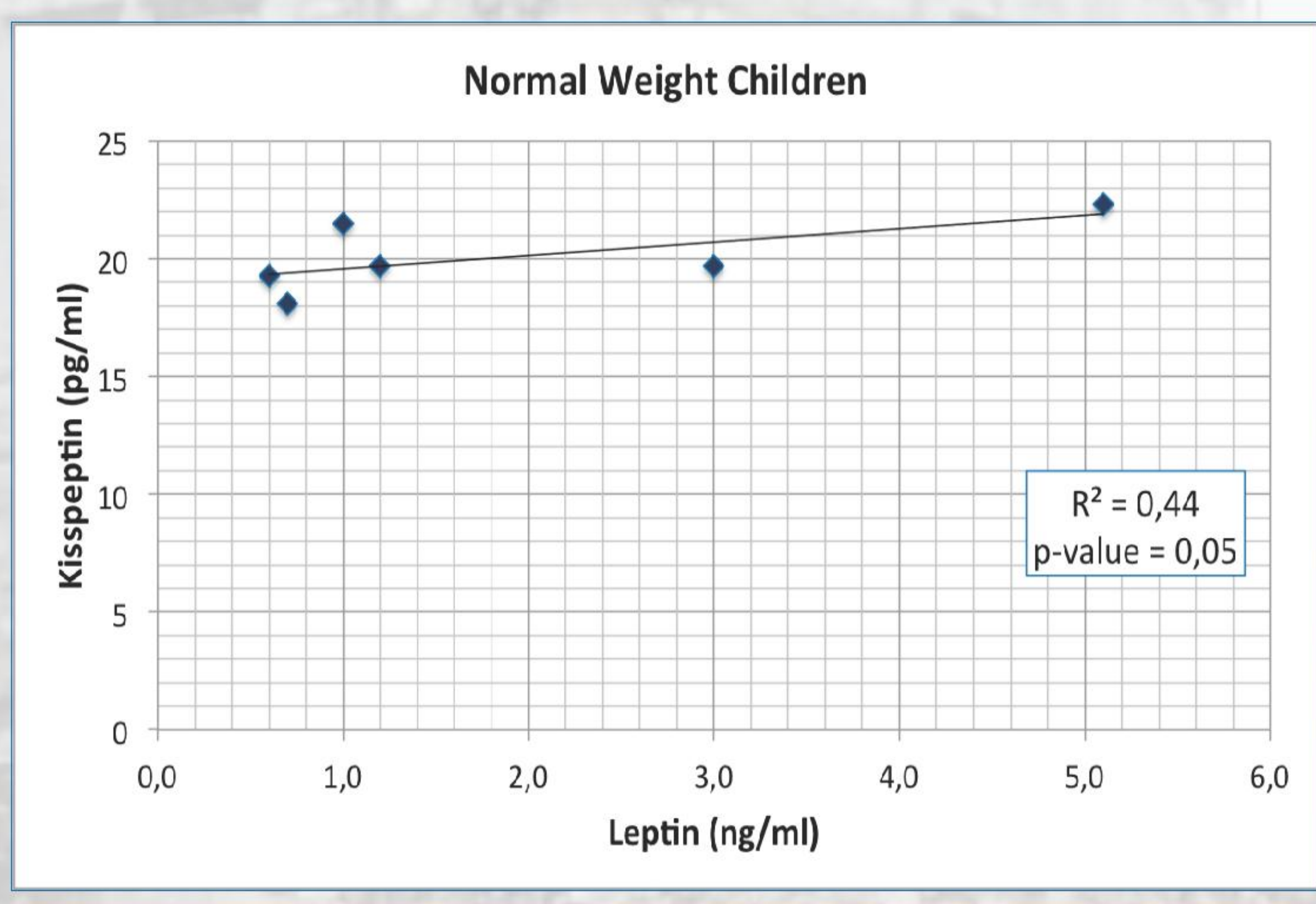

Fig. 1

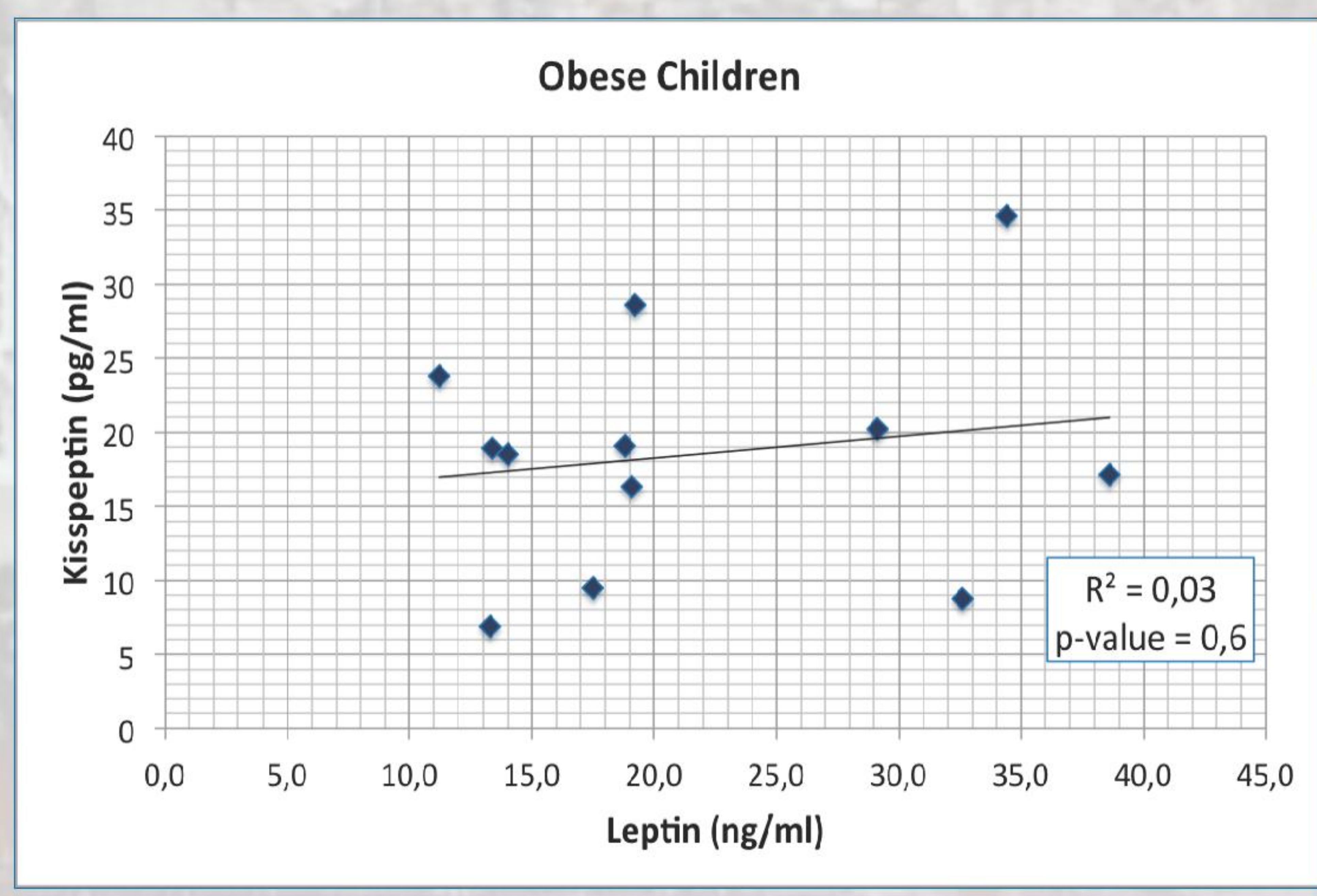

Fig. 2

\section{Conclusions}

In our population of obese children we have not find sex related differences, suggesting that the modulation of Kisspeptin in adult obese is acquired after puberty and linked to circulating sexual steroids.

An unexpected datum was the lack of correlation with Leptin levels in obese children; a larger number of subjects need to be examined to confirm this observation and investigate a possible metabolic role of Kisspeptin. 